\title{
THE INFRARED SPECTRUM OF P CYGNI
}

\author{
J. R. Deacon and M. J. Barlow \\ Department of Physics $\&$ Astronomy \\ University College London \\ Gower Street, London WC1E 6BT
}

\begin{abstract}
An energy distribution for $\mathrm{P}$ Cyg $\left(\mathrm{B}_{1 \mathrm{Ia}^{+}}\right)$has been produced using UKIRT photometry and spectra, flux-calibrated IUE high-resolution spectra, Johnson and Mitchell 13-colour photometry and IRAS photometry. Infrared excesses due to free-free emission from the wind have then been derived and modelled.
\end{abstract}

\section{The Infrared Excesses}

The IR excesses were derived by fitting a Kurucz model atmosphere to the UV and optical data, the best fit being given by a model with $T_{\text {eff }}=18000 \mathrm{~K}$ and $\log g=2.05$. Subtracting this model from the observations gave the excess fluxes, which have been modelled assuming a spherically symmetric wind parameterised by either Castor and Lamers (CL) velocity laws of index $\beta$ or by the linear velocity law used by Waters and Wesselius (1986, Astr. Astrophys., 155, 104). A full treatment of electron scattering and free-free and bound-free opacity was included, along with the radial dependence of wind electron temperature given by the work of Drew (1989, Astrophys. J. Suppl., 71, 267).

\section{Results}

A good fit to the observations was found using the linear velocity law with the following parameters :

$$
\begin{array}{llll}
T_{\text {eff }}=18000 \mathrm{~K} & \log g=2.05 & R_{*}=92.5 \mathrm{R}_{\odot} & D=1.8 \mathrm{kpc} \\
v_{\infty}=206 \mathrm{~km} \mathrm{~s}^{-1} & v\left(R_{*}\right) / v_{\infty}=0.175 & \dot{M}=2.16 \times 10^{-5} M_{\odot} y^{-1} & n(\mathrm{He}) / n(\mathrm{H})=0.5
\end{array}
$$

the value of $v_{\infty}$ is taken from Lamers et al. (1985, Astr. Astrophys., 149, 29), $\dot{M}$ from radio observations of van den Oord $(1985$, in Radio Stars $), n(\mathrm{He}) / n(\mathrm{H})$ was derived from the $J H K L$ spectra and $R_{*}$ by normalising the Kurucz model to the $J$ band magnitude.

\section{Discussion}

It was found that the linear velocity law gave the best fit to the observations. The CL velocity laws predicted energy distributions which fell off too steeply longward of $\sim 10 \mu \mathrm{m}$. It appears that longward of $\sim 25 \mu \mathrm{m}$ the adopted model starts to predict smaller excesses than are observed-the possible need to include density fluctuations such as those predicted by Owocki et al. (1988, Astrophys. J., 335, 914) will be investigated. 